\title{
A DIRECT SPECTRAL PROBLEM FOR $L$-SPECTRUM OF THE PERTURBED OPERATOR WITH A MULTIPLE SPECTRUM
}

E. V. Kirillov ${ }^{1}$, kirillovev@susu.ac.ru,

G. A. Zakirova ${ }^{1}$, zakirovaga@susu.ac.ru.

${ }^{1}$ South Ural State University, Chelyabinsk, Russian Federation

We consider a direct spectral problem for an operator having a non-nuclear resolvent and perturbed by the bounded operator with multiple spectrum. A similar problem was considered earlier for an operator with a single spectrum. The method of regularized traces is used as a method of solution. This method can not be applied directly to the problem. We propose to introduce the relative resolvent of the operator. A spectral problem of the form $(M+P) u=L u$ is obtained. In this case, the operator $L$ is such that the relative resolvent of the operator is a nuclear operator. As a result of applying the resolvent method to the relative spectrum of the perturbed operator, we obtain relative eigenvalues of the perturbed operator with non-nuclear resolvent.

Keywords: perturbed operator, discrete self-adjoint operator, direct spectral problem, relative resolvent, multiple spectrum.

\section{Introduction}

The theory of regularized traces is based on the following fundamental result of the linear algebra. A matrix trace of operator does not depend on the choice of basis and coincides with the spectral trace. It is proved that this result is true for a certain class of operators in Hilbert spaces [1].

Consider the remaining classes of operators, which have no trace. This means that such classes of operators are not nuclear, and therefore a series of their eigenvalues converges. For such classes of operators we search for pairs of basises such that the following relation holds:

$$
\sum_{n=1}^{\infty}\left[\left(A \varphi_{n}, \varphi_{n}\right)-\left(A \psi_{n}, \psi_{n}\right)\right]=0
$$

Therefore, we need to specify the class of operators and the corresponding pairs of basises for which this relation holds. We choose a basis from eigenvectors of the operator, and split the operator into the sum of two operators: $A=A_{0}+B$. Here the operator $B$ depends on the operator $A$ in some sense. The problem takes the following form:

$$
\begin{gathered}
\sum_{n=1}^{\infty}\left[\left(A \varphi_{n}, \varphi_{n}\right)-\left(A \psi_{n}, \psi_{n}\right)\right]= \\
=\sum_{n=1}^{\infty}\left[\left(\left(A_{0}+B\right) \varphi_{n}, \varphi_{n}\right)-\left(\left(A_{0}+B\right) \psi_{n}, \psi_{n}\right)\right]= \\
=\sum_{n=1}^{\infty}\left[\left(A \varphi_{n}, \varphi_{n}\right)-\left(\left(A_{0}+B\right) \psi_{n}, \psi_{n}\right)+\left(B \varphi_{n}, \varphi_{n}\right)\right]=
\end{gathered}
$$




$$
=\sum_{n=1}^{\infty}\left[\lambda_{n}-\mu_{n}+\left(B \varphi_{n}, \varphi_{n}\right)\right]=0 .
$$

The following formula of the trace

$$
\lim _{m \rightarrow \infty} \sum_{i=0}^{n_{i}}\left(\mu_{i}-\lambda_{i}-\left(B \varphi_{i}, \varphi_{i}\right)\right)=0
$$

is proved for operators having a nuclear resolvent and perturbed by the bounded operator $B$ [2]. The cases of relatively compact perturbation and non-nuclear operator were studied in [3]: if there exist a number $\delta \in[0,1)$ such that the operator $B A^{-1}$ extends to a bounded operator, and a number $\omega \in[0,1), \omega+\delta<1$ such that $A^{-(1-\delta-\omega)}$ is a nuclear operator, then there exists a subsequence of numbers $\left\{n_{m}\right\}_{m=1}^{\infty}$ such that for $\omega \geq \frac{\delta}{l}$

$$
\lim _{m \rightarrow \infty}\left(\sum_{j=0}^{n_{m}}\left(\mu_{j}-\lambda_{j}\right)+\frac{1}{2 \pi i} \int_{\Gamma_{m}} \sum_{k=1}^{l} \frac{(-1)^{k-1}}{k} \operatorname{Tr}\left(\left(B R_{0}(\lambda)\right)^{k}\right) d \lambda\right)=0 .
$$

Similar results were obtained in the case, when there exist the "large" gaps in the spectrum of the operator $A$.

In this paper we use the following resolvent method. Decompose resolvent of the perturbed operator into a series depending on resolvent of the unperturbed operator and the perturbation operator. Then integrate along a special contour and obtain an equality connecting the eigenvalues of the perturbed operator and the unperturbed operator. Advantage of the method is simplicity. Also, it is easy to check the required conditions for operators. The disadvantage is that the norm of the perturbation operator must be sufficiently small. The resolvent method is effective for direct and inverse spectral problems of the form

$$
(M+P) u=\lambda u,
$$

where the operator $M$ is linear and selfadjoint, and the perturbation operator $P$ is linear and bounded. Hereinafter, the condition for the boundedness of the perturbation operator is replaced by the condition that the operator depends on the operator $M$.

A direct and inverse spectral problem of the type

$$
M u=\lambda L u
$$

for an operator with a single spectrum was first considered in the papers [4], [5]. The direct spectral problem for one-dimensional Dzeczer equation was solved in [4]. The main result of the paper is the next theorem.

Theorem 1. Let $M=\alpha \Delta-\beta \Delta^{2}, \quad \Delta=\frac{d^{2}}{d x^{2}}, \quad L=a^{2}-\Delta$, and

$$
\begin{gathered}
F=W_{2}^{k}(0, \pi), k \in \mathbb{N} \bigcup\{0\}, \\
U=\left\{W_{2}^{k+2}(0, \pi): u(0)=u(\pi)=0\right\}, \\
\operatorname{dom} M=\left\{u \in W_{2}^{k+2}(0, \pi): u^{\prime \prime}(0)=u^{\prime \prime}(\pi)=0\right\} \bigcap U .
\end{gathered}
$$


Let $P$ be an operator of multiplication by, in general, the complex-valued function $p \in$ $C^{2}(0, \pi)$, satisfying the following condition:

$$
p^{\prime}(0)=p^{\prime}(\pi) \text {. }
$$

Consider the operator $M+P$. Denote $\left\{\nu_{n}\right\}_{n=1}^{\infty}=\sigma^{L}(M+P)$, where $\nu_{n}$ are numbered in order of nonincreasing of their real parts, taking into account the algebraic multiplicity.

If the conditions

1. $p^{\prime}(0)=p^{\prime}(\pi)$,

2. $\|P\|<\frac{\beta}{2}$,

3. $p(x) \in C_{[0, \pi]}^{2}$

hold, then the following formula for the relative regularized trace of the perturbed operator $M+P$ is true:

$$
\sum_{n=1}^{\infty}\left[\nu_{n}-\mu_{n}-\frac{2}{\pi\left(a^{2}-\lambda_{n}\right)} \int_{0}^{\pi} p(x) d x\right]=0 .
$$

The spectral problems for the Dzieczer equation were considered in [6]. The problems were solved using the Galerkin method. Let us note the main results of the paper.

Theorem 2. Let operators $M, L: U \rightarrow F$ be defined by the formulas

$$
M=\alpha \Delta-\beta \Delta^{2}, \Delta=\frac{d^{2}}{d x^{2}}, L=a^{2}-\Delta
$$

and

$$
\begin{gathered}
U=\left\{u \in W_{2}^{k+2}(0, b): u(x)=0, x \in(0, b)\right\}, \\
F=W_{2}^{k}(a, b), k \in\{0\} \bigcup \mathbb{N}, u=W_{2}^{k+2}(0, b), \\
\operatorname{dom} M=\left\{u \in W_{2}^{k+2}(0, b): u^{\prime \prime}(0)=u^{\prime \prime}(b)=0\right\} \bigcup U .
\end{gathered}
$$

Let operator $P$ be an operator of multiplication by the function $p \in C^{2}(0, b)$. If the system of functions $\left\{\varphi_{k}\right\}_{k=1}^{\infty}$ is a basis in $H$, then the Galerkin method, which is applied to the spectral problem $(M+P) u=\nu$ Lu of finding the eigenvalues, converges.

The following formula holds for the inverse spectral problem (recovery of the potential):

$$
\tilde{p}=\alpha_{0}-\sqrt{4 a b}\left(\sum_{j \neq t} \sum_{k=1}^{\nu_{t}} \frac{\left(\alpha_{0} \varphi_{t}, \varphi_{j}\right)^{2}}{\left(\lambda_{t}-\lambda_{j}\right)}\right) \varphi_{t}^{k} .
$$

Direct and inverse spectral problems play an important role to solve the problems of hydra dynamical stability, as well as to consider vibrations of a membrane and elastic bodies in the shell theory, in the quantum mechanics, to study an electrical oscillations in a long-distance line, in the geophysical models of the terrestrial sphere, in the cosmology. The resolvent method allows effectively solve such problems, if the perturbed operator has a nuclear resolvent and is perturbed by a bounded operator. Note that this method is extremely difficult for an operator with a non-nuclear resolvent, because in this case we need to search for a special function that transforms eigenvalues of the operator. It is possible to solve problems with an unbounded perturbation operator, but the operator 
must be in some sense depended on the perturbed operator. The paper is devoted to the study of the direct spectral problem for an operator with a multiple spectrum whose resolvent is not a nuclear operator. A similar problem for an operator with a single spectrum was considered in the paper [7]. In order to solve the considered problem, the resolvent method developed by V.A. Sadovnichy and V.V. Dubrovsky [8] is applied to the relative or $L$-resolvent of the operator [9]. In the paper [4] this approach was applied to the operator with nuclear resolution.

\section{Necessary Statements}

Let $M$ be a discrete, selfadjoint, positive, semi-bounded from below operator, $L$ be a linear, closed, continuously invertible operator. The operators $M$ and $L$ act in the separable Hilbert space $\mathfrak{H}$. Let $P$ be a bounded operator acting in the same space $\mathfrak{H}$. Denote $L$-resolvent of the operator $M$ by $R_{0}(\mu)=(\mu L-M)^{-1}$, and $L$-resolvent of the perturbed operator $M+P$ by $R(\mu)=(\mu L-M-P)$. Let $\left\{\lambda_{n}\right\}_{n=1}^{\infty}$ be eigenvalues of the operator $M$ numbered in order of nonincreasing of their real parts, taking into account the algebraic multiplicity, $\left\{\mu_{n}\right\}_{n=1}^{\infty}=\partial^{L}(M)$ be $L$-spectrum of the operator $M,\left\{\nu_{n}\right\}_{n=1}^{\infty}=\partial^{L}(M+P)$ be $L$-spectrum of the operator $M+P$. The eigenvalues of the operator $M+P$ are numbered in order of nonincreasing of their real parts, taking into account the algebraic multiplicity.

Lemma 1. [7] If $\|P\|<r / 2$, where $0<r \leq r_{0}$, then the operator $M+P$ is discrete, and

(i) if $R_{0}(\lambda) \in \mathfrak{S}_{q}$, then $R(\lambda) \in \mathfrak{S}_{q}, 1 \leq q<\infty$,

(ii) if $\lambda_{n} \in \mathbb{C} \backslash \Omega_{r_{n}}$, then $\mu_{n}^{s} \in \mathbb{C} \backslash \Omega_{r_{n}}, s=\overline{1, \eta_{n}}, \eta_{n}$ is multiplicity of the eigenvalue $\lambda_{n}$.

The proof of the following lemmas repeats the proof proposed in the paper [5] and does not change for a multiple and a single spectrum of the operator $M$.

Lemma 2. [7] If for $\mu \in \gamma_{n}\left\|P R_{0}(\mu)\right\|=q<1$, then the following equality holds:

$$
L R(\mu)=L R_{0}(\mu)+\sum_{k=1}^{\infty}\left[R_{0}(\mu) P\right]^{k} L R_{0}(\mu) .
$$

Lemma 3. [7] Let $\mu \in \rho^{L}(M)$, then the following estimate holds:

$$
\left\|L R_{0}(\mu)\right\| \leq \frac{1}{\rho\left(\mu, \sigma^{L}(M)\right)}
$$

where $\rho\left(\mu, \sigma^{L}(M)\right)$ is a distance between the point $\mu$ and L-spectrum of the operator $M$.

Theorem 3. Let $M$ be a discrete, selfadjoint, positive, semibounded from below operator, $L$ be a linear, closed, continuously invertible operator such that $L^{-1}$ is a bounded operator, $R_{0}(\mu)$ be a nuclear operator and $P \leq \frac{r_{0}}{2}$, then there is a spectral identity:

$$
\sum_{q=1}^{\eta_{n}} \nu_{n}^{q}=\eta_{n} \mu_{n}+\sum_{q=1}^{\eta_{n}}\left(L^{-1} P \varphi_{n}, \varphi_{n}\right)+\alpha_{n}
$$

where

$$
\alpha_{n}=\sum_{k=2}^{\infty} \frac{(-1)^{k}}{2 \pi i} \int_{\gamma_{n}} \mu S p\left[R_{0}(\mu) P\right]^{k} L R_{0}(\mu) d \mu .
$$


Proof.

Consider the series (5)

$$
L R(\mu)=L R_{0}(\mu)+\sum_{k=1}^{\infty}\left[R_{0}(\mu) P\right]^{k} L R_{0}(\mu) .
$$

Multiply the right and left sides of this equality by $\frac{\mu}{2 P i i}$ and integrate the resulting equation along the contour $\gamma_{n}$. We have

$$
\frac{1}{2 \pi i} \int_{\gamma_{n}} \mu L R(\mu) d \mu=\frac{1}{2 \pi i} \int_{\gamma_{n}} \mu L R_{0}(\mu) d \mu+\sum_{k=1}^{\infty} \frac{1}{2 \pi i} \int_{\gamma_{n}} \mu\left[R_{0}(\mu) P\right]^{k} L R_{0}(\mu) d \mu .
$$

In order to search for the matrix trace of both sides of the obtained equality we use nuclearity of the operators $T$ and $T+P$.

$$
\begin{gathered}
S p \frac{1}{2 \pi i} \int_{\gamma_{n}} \mu L R_{0}(\mu) d \mu=S p \frac{1}{2 \pi i} \int_{\gamma_{n}} \mu \sum_{k=1}^{\infty} \frac{P_{k}}{\mu-\mu_{k}} d \mu=\sum_{q=1}^{\eta_{n}} \mu_{n}^{q} S p P_{n}=\sum_{q=1}^{\eta_{n}} \mu_{n}^{q} \sum_{s=1}^{\infty}\left(P_{n} \varphi_{s}^{q}, \varphi_{s}^{q}\right)= \\
=\sum_{q=1}^{\eta_{n}} \mu_{n}^{q} \sum_{s=1}^{\infty}\left(\left(\varphi_{s}^{q}, \varphi_{n}\right) \varphi_{n}, \varphi_{s}^{q}\right)=\sum_{q=1}^{\eta_{n}} \mu_{n}^{q} \sum_{s=1}^{\infty}\left(\left(\varphi_{s}^{q}, \varphi_{n}\right)\left(\varphi_{n}, \varphi_{s}^{q}\right)\right)=\sum_{q=1}^{\eta_{n}} \mu_{n}=\eta_{n} \mu .
\end{gathered}
$$

Similarly,

$$
S p \frac{1}{2 \pi i} \int_{\gamma_{n}} \mu L R(\mu) d \mu=\sum_{q=1}^{\eta_{n}} \nu_{n}^{q}
$$

Calculate the first correction of the perturbation theory:

$$
\begin{gathered}
\frac{1}{2 \pi i} \int_{\gamma_{n}} S p \mu R_{0}(m u) P L R_{0}(\mu)=\frac{1}{2 \pi i} \int_{\gamma_{n}} \mu \sum_{s=1}^{\infty}\left(R_{0}(\mu) P L R_{0}(\mu) \varphi_{s}, \varphi_{s}\right) d \mu= \\
=\frac{1}{2 \pi i} \int_{\gamma_{n}} \mu \sum_{s=1}^{\infty}\left(\frac{1}{\mu-\mu_{s}} L^{-1} P L \frac{1}{\mu-\mu_{s}} L^{-1} \varphi_{s}, \varphi_{s}\right) d \mu= \\
=\frac{1}{2 \pi i} \int_{\gamma_{n}} \mu \sum_{s=1}^{\infty}\left(\frac{1}{\left(\mu-\mu_{s}\right)^{2}} L^{-1} P L L^{-1} \varphi_{s}, \varphi_{s}\right) d \mu= \\
=\frac{1}{2 \pi i} \sum_{s=1}^{\infty} \int_{\gamma_{n}} \mu \frac{1}{\left(\mu-\mu_{s}\right)^{2}}\left(L^{-1} P \varphi_{s}, \varphi_{s}\right) d \mu= \\
=\sum_{q=1}^{\eta_{n}}\left(L^{-1} P \varphi_{n}^{q}, \varphi_{n}^{q}\right) .
\end{gathered}
$$

We obtain the following spectral identity:

$$
\sum_{q=1}^{\eta_{n}} \nu_{n}^{q}=\eta_{n} \mu_{n}+\sum_{q=1}^{\eta_{n}}\left(L^{-1} P \varphi_{n}^{q}, \varphi_{n}^{q}\right)+\frac{1}{2 \pi i} \sum_{k=2}^{\infty} \int_{\gamma_{n}} \mu\left(R_{0}(\mu) P\right)^{k} L R_{0}(\mu) .
$$


Show that $\alpha_{n} \rightarrow 0$ for $n \rightarrow \infty$. Consider

$$
\alpha_{n}=\sum_{k=2}^{\infty} \frac{(-1)^{k}}{2 \pi i} \int_{\gamma_{n}} \mu \operatorname{Sp}\left[R_{0}(\mu) P\right]^{k} L R_{0}(\mu) d \mu=\sum_{k=2}^{\infty} \frac{(-1)^{k+1}}{2 \pi i k} \int_{\gamma_{n}} \mu \operatorname{Sp}\left[R_{0}(\mu) P\right]^{k} L R_{0}(\mu) d \mu .
$$

The main idea of the further proof is based on the paper [2]. Estimate terms of the series for $k \geq 2$. Denote the distance between the point $\mu \in \mathbb{C}$ and the relative spectrum $T$ by $d(\mu)$.

$$
\begin{gathered}
\int_{\gamma_{n}}\left|\operatorname{Sp}\left[P R_{0}(\mu)\right]^{k} L R_{0}(\mu) \| d \mu\right| \leq \\
\leq \int_{\gamma_{n}}\left\|P R_{0}(\mu)\right\|^{k-1}\|P\|\left\|R_{0}(\mu)\right\|_{1}\left\|L R_{0}(\mu)\right\| d \mu \| \leq \\
\leq\|P\|^{k}\left\|R_{0}(\mu)\right\|_{1} \int_{\gamma_{n}} \frac{|d \mu|}{d^{k-1}(\mu)}\left\|L R_{0}(\mu)\right\| .
\end{gathered}
$$

Estimate the integral $\int_{\gamma_{n}} \frac{|d \mu|}{d^{k-1}(\mu)}$ in the first quarter of the circle having radius $\frac{r_{n}}{2}$ with center at the point $\mu_{n}$ (in the remaining quarters similarly). Let $\mu=r e^{i \beta}$, then $d(\mu) \geq r \sin \beta$ and on the contour $\gamma_{n} d(\mu)=\frac{r_{n}}{2}$.

$$
\begin{gathered}
\int_{\gamma_{n}} \frac{|d \mu|}{d^{k-1}(\mu)}=\int_{0}^{\theta} \frac{r\left|e^{i \beta}\right| d \beta}{d^{k-1}\left(r e^{i} \beta\right)} \leq \\
\left.\leq \int_{0}^{\theta} \frac{r d \beta}{r \sin \beta d^{k-2}\left(r e^{i \beta}\right)}=\int_{0}^{\theta} \frac{d \beta}{\sin \beta d^{k-2}\left(r e^{i \beta}\right)}=\int_{0}^{\theta} \frac{d \beta}{\sin \beta\left(\frac{r_{n}}{2}\right)^{k-2}}\right) \leq \frac{\text { const }}{\left(\frac{r_{n}}{2}\right)^{k-2}},
\end{gathered}
$$

where $\theta=\frac{r_{n}}{2 \sqrt{\frac{r_{n}^{2}}{4}+\mu_{n}^{2}}}$. We obtain that

$$
\begin{gathered}
\left|\sum_{k=2}^{\infty} \frac{(-1)^{k+1}}{2 \pi i k} \int_{\gamma_{n}} \mu \operatorname{Sp}\left[R_{0}(\mu) P\right]^{k} L R_{0}(\mu) d \mu\right| \leq \\
\leq \text { const }\left\|R_{0}\left(r_{n}\right)\right\|_{1} \sum_{k=2}^{\infty} \frac{\|P\|^{k}}{\left(\frac{r_{n}}{2}\right)^{k-2}}\left\|L R_{0}(\mu)\right\| \leq \text { const }\left\|R_{0}\left(r_{n}\right)\right\|_{1}\left\|L R_{0}\left(r_{n}\right)\right\| .
\end{gathered}
$$

\section{References}

1. Lidskiy V.B. The Non-Self-Adjoint Operators Having a Trace. Dokl. AN SSSR, 1959, vol. 125, no. 3, pp. 485-487. (in Russian).

2. Sadovnichii V.A., Konyagin S.V., Podol'skii V.E. The Regularized Trace of an Operator Having a Nuclear Resolvent and Perturbed by a Bounded Operator. Doklady Mathematics, 2000, vol. 62, no. 1, pp. 19-21. (in Russian).

3. Sadovnichii V.A., Podolskii V.E. Traces of Operators with Relatively Compact Perturbations. Sb. Math., 2002, vol. 193, no. 2, pp. 279-302. (in Russian). doi: $10.4213 / \mathrm{sm} 630$.

4. Zakirova G.A., Kirillov E.V. L-regularized Trace of One of the Perturbed Operator. Bulletin of the Odessa National University. Mathematics and Mechanics, 2013, vol. 18, issue 2(18), pp. 7-13. (in Russian). 
5. Kirillov E.V. The Spectral Identity for the Operator with Non-Nuclear Resolvent Journal of Computational and Engineering Mathematics, 2017, vol. 4, no. 1, pp. 69-75. doi: $10.14529 /$ jcem170107.

6. Strepetova I.S., Fatkullina L.M., Zakirova G.A. Spectral Problems for One Mathematical Model of Hydrodynamics Journal of Computational and Engineering Mathematics, 2017, vol. 4, no. 1, pp. 48-56. doi: 10.14529/jcem170105.

7. Zakirova G.A., Sedov A.I. An Inverse Spectral Problem for Laplace Operator and It's Approximate Solution. Bulletin of the South Ural State University. Series: Mathematical Modelling, Programming and Computer Software, 2008, no. 27(127), issue 2, pp. 19-27. (in Russian).

8. Sadovnichy V.A., Dubrovskii V.V. An Abstract Theorem of Perturbation Theory, Regularized Trace Formulas and Zeta Function Operator. Differ. Uravn., 1977, vol. 13, no. 7, pp. 1264-1271. (in Russian).

9. Sviridyuk G.A., Fedorov V.E. Linear Sobolev Type Equations and Degenerate Semigroups of Operators. Utrecht, Boston, VSP, 2003.

Evgenii V. Kirillov, Postgraduate Student, Department of Equations of Mathematical Physics, South Ural State University (Chelyabinsk, Russian Federation), kirillovev@susu.ac.ru.

Galiya A. Zakirova, Candidate of Physico-Mathematical Sciences, Docent, Department of Equations of Mathematical Physics, South Ural State University (Chelyabinsk, Russian Federation), zakirova81@mail.ru.

Received September 2, 2017

УДК 517.9

DOI: $10.14529 /$ jcem 170303

\section{ПРЯМАЯ СПЕКТРАЛЬНАЯ ЗАДАЧА ДЛЯ $L$-СПЕКТРА ВОЗМУЩЕННОГО ОПЕРАТОРА С КРАТНЫМ CПЕКТРОМ}

\section{Е. В. Кириллов, Г. А. Закирова}

В данной работе рассматривается прямая спектральная задача для оператора с нядерной резольвентой возмущенного ограниченным оператором, имеющим кратный спектр. Похожая задача рассматривалась ранее для оператора с однократным спектром. В качестве метода решения используется метод регуляризованных следов. На прямую этот метод применить к данной задаче не удается. Предлагается ввести в рассмотрение относительную резольвенту оператора. Получаем спектральную задачу вида $(M+P) u=L u$. При этом оператор $L$ выбран таким образом, что относительная резольвента оператора является ядерным оператором. В результате применения резольвентного метода к относительному спектру возмущенного оператора получаем относительные собственные числа возмущенного оператора с неядерной резольвентой.

Ключевые слова: возмущенный оператор, дискретный самосопряженный оператор, потенииал, кратный спектр 


\section{Литература}

1. Лидский, В.Б. Несамосопряженные операторы, имеющие след / В.Б. Лидский // Докл. АН СССР. - 1959. - Т. 125, № 3. - С. 485-487.

2. Садовничий, В.А. Регуляризованный след оператора с ядерной резольвентой, возмущенного ограниченным / В.А. Садовничий, С.В. Конягин, В.Е. Подольский // Доклады Академии наук. - 2000. - Т. 62, № 1. - С. 19-21.

3. Садовничий, В.А. Следы операторов с относительно компактным возмущением / В.А. Садовничий, В.Е. Подольский // Матем. сб. - 2002. - Т. 193, № 2. C. $129-152$.

4. Закирова, Г.А. Регуляризованный $L$-след одного возмущенного оператора / Г.А. Закирова, Е.В. Кириллов // Вестник Одесского национального университета. Математика и механика. - 2013. - Т. 18. Выпуск 2(18). - С. 7-13.

5. Kirillov, E.V. The spectral identity for the operator with non-nuclear resolvent E.V. Kirillov // Journal of Computational and Engineering Mathematics. - 2017. V. 4, №. 1. - P. 69-75.

6. Strepetova, I.S. Spectral problems for one mathematical model of hydrodynamics / I.S. Strepetova, L.M. Fatkullina, G.A. Zakirova // Journal of Computational and Engineering Mathematics. - 2017. - V. 4, №. 1. - P. 48-56.

7. Закирова, Г.А. Обратная спектральная задача для оператора Лапласа и ее приближенное решение / Г.А. Закирова, А.И. Седов // Вестник Южно-Уральского государственного университета. Серия: Математическое моделирование и программирование. - 2008. - № 27(127), вып. 2. - С. 19-27.

8. Садовничий, В.А. Об одной абстрактной теореме теории возмущений, о формулах регуляризованных следов и о дзета-функции операторов / В.А. Садовничий, В.В. Дубровский // Дифференц. уравнения. - 1977. - Т. 13, № 7. - С. 1264-1271.

9. Sviridyuk, G.A. Linear sobolev type equation and degenerator semigroups of operators. / G.A. Sviridyuk, V.E. Fedorov // Utrecht, Boston: VSP. - 2003.

Кириллов Евгений Вадимович, аспирант, кафедра уравнений математической физики, Южно-Уральский государственный университет (г. Челябинск, Российская Федераuия),kirillovev@susu.ac.ru

Закирова Галия Амрулловна, кандидат физико-математических наук, дочент, кафедра уравнений математической физики, Южно-Уральский государственный университет (2. Челябинск, Российская Федерация), zakirovaga@susu.ru

Поступила в редакиию 2 сентября 2017 г. 